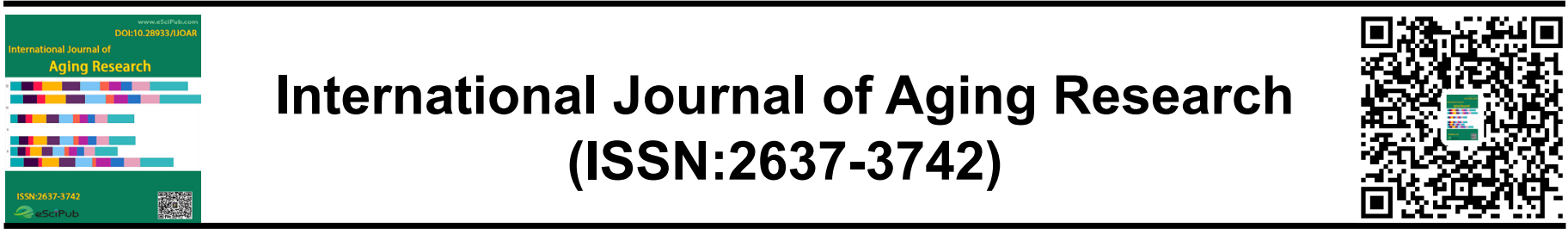

\title{
NEW APPROACHER IN THE DEVELOPMENT OF ALZHEIMER'S DISEASE MODIFYING DRUGS
}

\author{
Pedro César de Souza ${ }^{1 *}$, Emmilli Emmanuelle Souza Lins Lima de Carvalho, Patrícia Maria \\ da Silva Neri Cruz ${ }^{2}$ \\ ${ }^{1}$ University Center Mauricio de Nassau, Recife, PE, Brasil; ${ }^{2}$ Federal University of Pernambuco, \\ Recife, PE, Brasil.
}

\begin{abstract}
Introduction: Alzheimer's disease is a more common neurodegenerative disease, affecting 25 million people worldwide, or accounting for about 60 to $70 \%$ of all dementia cases. There is currently no exact mechanism to explain the pathophysiology of Alzheimer's disease, however, cascading metabolic amyloid and post-translational review of tau protein are used as major hypotheses. Objective: To demonstrate in the literature new approaches in the development of Alzheimer's disease modifiers. Methodology: For the accomplishment of this study made in the bibliographical survey of scientific literature and respect to the approached subject, in the databases PUBMED, ScienceDirect, Scielo and Scopus. Results: Alzheimer's disease-modifying drugs are not yet available, but many patients may, however, develop phase III clinical trials and are intended to modify as pathological stages leading to the disease. As disease-modifying therapies under study, these changes also affect $A \beta$ and tau protein and also cause inflammation and oxidative damage. The results obtained in the clinical trials performed were positive and promising and are still under study. The results show that there is still a long way to go in the development of Alzheimer's disease modifying drugs. Conclusion: The results demonstrated that there is still a long way to go in the development of Alzheimer's disease modifying drugs, but nevertheless levels at the research level should be continued in order to improve the pathophysiolo${ }^{*}$ Correspondence to Author: Pedro César de Souza University Center Mauricio de Nassau, Recife, PE, Brasil;

How to cite this article:

Pedro César de Souza, Emmilli Emmanuelle Souza Lins Lima de Carvalho, Patrícia Maria da Silva Neri Cruz. NEW APPROACHER IN THE DEVELOPMENT OF ALZHEIMER'S DISEASE MODIFYING DRUGS. International Journal of Aging Research, 2021, 4:88 gy of the disease and find an effective treatment for this disease the same.
\end{abstract}

Keywords: Alzheimer's disease, $\beta$-amyloid, Tau hyperphosphorylation, Neurodegeneration 


\section{INTRODUCTION}

Neurodegenerative diseases are diseases that affect the central nervous system (CNS) causing progressive degeneration and neuronal death in certain brain regions ${ }^{[1]}$. There are several neurodegenerative diseases such as Alzheimer's disease (AD), Parkinson's disease, Multiple Sclerosis, Lewy body disease, among others ${ }^{[2]}$. AD is the most common neurodegenerative disease affecting about 25 million people worldwide, accounting for about $60-70 \%$ of all dementia cases. These values are expected to triple by $2050{ }^{[3,4]}$. The main neuropathological changes in $A D$ are cerebral atrophy, the presence of brain senile plaques containing extracellular deposits of the $\beta$ amyloid peptide $(A \beta)$, intraneuronal neurofibrillary tangles (ENF) containing the hyperphosphorylated tau protein, and loss of neuronal cells. These neuropathological changes manifest with the progressive loss of memory and cognitive functions (difficulty in reasoning, disorientation, and difficulties in expressing themselves. ${ }^{[5,6]}$ There is currently no exact mechanism to explain the pathophysiology of $A D$, however the metabolic cascade. amyloid and post-translational modification of tau protein, the protein responsible for stabilizing microtubules, are considered the most important hypotheses, and other mechanisms have been considered as neurotransmitter dysfunction, mitochondrial dysfunction, oxidative stress, genetic and environmental factors ${ }^{[7]}$. Current treatments available for $A D$ do not alter disease progression, are limited to the alleviation of cognitive, behavioral and psychological symptoms. The two approved drug classes for $A D$ treatment are: acetylcholinesterase inhibitors (IAChE) and receptor antagonist. Nmethyl-D-aspartate glutamate (NMDA) IAChE are the drugs used as first-line treatment for $A D$ and are donepezil, rivastigmine and galantamine. In a later stage of the disease, memantine is used, which is the only noncompetitive NMDA receptor antagonist approved for the treatment of $A D$. In order to treat the behavioral symptoms of the disease, antipsychotics, antidepressants and benzodiazepines are also used simultaneously ${ }^{[3]}$. Several studies are under development in phase III clinical trials and are intended to modify the pathological stages leading to the disease ${ }^{[8]}$. The disease-modifying therapies under study have as their main targets $A \beta$ and tau protein as well as inflammation7.

\section{OBJECTIVE}

Demonstrate in the literature new approaches in the development of Alzheimer's disease modifying drugs

\section{METHODOLOGY}

For the accomplishment of this study made a bibliographical survey in the scientific literature about the proposed theme. Selection for this review was based on journals in the following databases: PUBMED (National Library of Medicine and The National Institute of Health), ScienceDirect, Scielo (Scientific Electronic Library Online) and Scopus. The following keywords will be searched for scientific articles: Alzheimer's disease, $\beta$-amyloid, Tau hyperphosphorylation, Neurodegeneration, with a 5-year temporal cut.

\section{RESULTS AND DISCUSSION}

Currently, drugs that are under development for $A D$ are intended to modify the pathological stages that lead to the disease, thus acting on the evolution of the disease and thus being called disease modifying drugs ${ }^{[8]}$. During the last decades, several hypotheses have been proposed for the pathogenesis of $A D$, with the theory of $\beta$-amyloid cascade $(A \beta)$ and tau hyperphosphorylation being the most widely accepted theories. Thus, the disease-modifying therapies under study have as their main targets $A \beta$ and tau protein, as well as inflammation ${ }^{[8,5]}$. There are three types of therapeutic approaches under development which are: secretinase inhibitors ( $\beta$ and $\gamma$ ), $\alpha$-secretase activators, inhibitors of $A \beta$ aggregation ${ }^{[4,5,8]}$. The BACE1 $\beta$ secretase enzyme is a promising therapeutic target, so a good BACE1 inhibitor must be 
metabolically stable, orally bioavailable, have low molecular weight, reduced susceptibility to P-glycoprotein or other carriers to achieve effective efficacy. BBB penetration. In addition they should also be highly selective so as not to interfere with other aspartic acid proteases (such as BACE2, cathepsin D) which have a high homology to the active site of $\mathrm{BACE}^{[5,2]}$. Nonpeptide $\beta$-secretase inhibitors are in clinical trials in animal models with the prospect of promising results $^{[9]}$. CTS-21166 is a $\beta$-secretase inhibitor that has entered a phase I clinical trial with healthy young people to assess the safety and preliminary response of $A \beta$. This assay has shown that this inhibitor has been shown to inhibit plasma $A \beta$. The next step will be to develop inhibitors with better pharmaceutical properties and to conduct clinical trials to evaluate efficacy in patients with $A D^{[10,11]}$. The development of $\mathrm{Y}$-secretase inhibitors is a promising target for $A D$ modification. $\Gamma$ secretase is a complex enzyme composed of four individual proteins which are preseniline, nicastrin, APH-1 (formerly pharynx-defective 1) and PSEN2 and has multiple cleavage sites and essential biological substrates. PPA is the best known substrate of $\mathrm{Y}$-secretase, whose cleavage produces $A \beta$. A group of non-steroidal anti-inflammatory drugs (NSAIDs) have been discovered to reduce $A \beta 42$ levels by modeling the $\mathrm{Y}$-secretase pathway ${ }^{[12]}$. Tarenflurbil (Flurizan®), which is a lurbiprofen NSAID Renantiomer, was the first modulator of $y$ secretase activity and acts by binding to a different site than the active center of the enzyme by altering the conformation of $\mathrm{Y}$ secretase and thus interfering with PPA cleavage, producing smaller and non-toxic $A \beta$ fragments without in any way altering other substrates essential to the enzyme. In studies in transgenic mice, tarenflurbil has been shown to reduce amyloid plaque accumulation, improve memory and behavioral performance ${ }^{[12]}$. Subsequently, clinical trials were conducted with subjects with mild to moderate $A D$, where in the phase I study the drug was well tolerated with no evidence of gastrointestinal toxicity but, however, did not produce a significant reduction in CSF and plasma A 342 . Phase II and phase III trials showed that there were positive effects on daily and global tasks, but did not reveal benefits on cognitive performance. These results questioned the benefits of $\gamma$-secretase modeling as well as the amyloid cascade hypothesis. One possible reason for the failure of this study is the poor potency of tarenflurbil as a $\mathrm{Y}$-secretase modeler and a poor distribution of this compound at the CNS leve[ ${ }^{[12]}$. Recently, second generation $\mathrm{Y}$-secretase modulators such as avagacestat (BMS-708), begacestat (GSI-953) and E2012 (highly selective Notch sparing inhibitor), begacestat ( GSI-953) and E201213. Avagacestat is a highly selective inhibitor of $\mathrm{y}$ secretase, which has already been tested in a phase I clinical trial and a decrease in CSF A $\beta$ has been observed. It is currently in a phase II clinical trial14. Another possible therapeutic approach is stimulation of the $\alpha$-secretase enzyme because since the $\alpha$-secretase and $\beta$ secretase enzymes compete for the same PPA substrate, if there is a greater activation of $\alpha$ secretase there will be a decrease in the SPPA $\beta$ substrate. available for the amyloidogenic pathway and thus a decrease in $A \beta$ production. Consequently there is a greater formation of soluble fragment sPPAa which have neuroprotective properties and is a stimulant of synaptogenesis. Thus, activation of $\alpha$-secretase has great therapeutic potential for $A D^{[15]}$. At this moment, there are not many compounds that stimulate this a-secretase pathway that have reached a phase of animal studies and later clinical trials with $A D$ patients such as etazolate ${ }^{[16]}$. Etazolate is an at disease than the amyloid burden. Thus, given the importance of this protein for $A D$, tau modification-based therapies are under investigation as an alternative target to $A \beta$ protein-based therapies. There is evidence from studies that tau aggregation can be disrupted by low molecular weight compounds such as methylthionine chloride (CMT) ${ }^{[18]}$. CMT, better known as methylene blue, is a promising compound as it has antioxidant properties, reduces $A \beta$ international-journal-of-aging-research/ 
oligomerization and interferes with tau aggregation by binding to the domain responsible for self-aggregating truncated tau fragments. A placebo-controlled phase II trial was performed in 321 subjects with mild or moderate AD using $30 \mathrm{mg}, 60 \mathrm{mg}$ or $100 \mathrm{mg}$ doses of CMT for 24 weeks 18 . With this study, an $80 \%$ decrease in $A D$ progression rate was observed in subjects receiving CMT compared with the placebo group. Despite the promising results observed, further studies are needed to demonstrate the efficacy and safety of CMT and a phase III clinical trial is pending approval19. Epothilone D (BMS-241027) is a microtubule stabilizing agent that acts by inhibiting microtubule disintegration to maintain the axon transport function, and on the other hand, prevents the formation of tau aggregates. Studies have been conducted in animal models with epothilone $D$ where there has been a decrease in cognitive and behavioral deficits. Epothilone D can penetrate BBB and exert better efficacy at low concentration and a phase I clinical trial is currently underway in individuals with $A D^{[19]}$. Nicotinamide is a NAD + coenzyme precursor compound that has been shown in animal model studies to reduce tau phosphorylation and to protect microtubule stabilization. This compound has been subjected to clinical trials with patients with mild to moderate $A D$ and has been shown to be safe and well tolerated so that a phase II clinical trial is currently under development ${ }^{[19]}$. Inflammatory phenomena occur on the periphery of amyloid plaques, through the accumulation of microglial cells around the plaques, cytokine reactions and activation of the complement cascade. Epidemiological evidence suggests that prolonged use of NSAIDs protects against the development of $A D$, and this protective effect is dependent on the duration of treatment [15,16]. However, several prospective studies with rofecoxib, naproxen, diclofenac, celecoxib, dapsone, hydroxychloroquine and nimesulin have shown that these drugs failed to slow the progression of cognitive decline in patients with mild to moderate AD. However, the same group of patients was used in an indomethacin study where cognitive decline was delayed, but due to the high gastrointestinal toxicity of indomethacin limited its use ${ }^{[20]}$.

\section{CONCLUSION}

The drugs currently approved for AD are IAChE and the NMDA glutamate receptor antagonist, however they do not alter disease progression, but only decrease the severity of its symptoms. Therefore, it is necessary to invest in scientific research in order to develop drugs that modify $A D$ by halting its progression. There are currently no $A D$ modifying drugs available, although several drugs have already been tested in phase III clinical trials, but none have yet been approved. Drugs such as tarenflurbil, tramiprosate and semagacestat were ineffective in the final clinical stages of the trials and other drugs such as avagacestat, etazolate, CAD-106, solanezumab, IVIg, methylthionine chloride and lithium are still being tested. in advanced clinical trials. In conclusion, it is of great importance to continue the investigation in order to broaden and deepen the understanding of the pathophysiology of $A D$, realizing a better relationship between $A \beta$ protein, tau protein and other factors that trigger the disease. Combination therapies of drugs with different targets or modifying factors (eg, $A \beta$, tau, and apoE4) are a hypothesis to consider. Further studies should be conducted to develop better disease biomarkers that will be useful in both diagnosis and clinical trials.

\section{References}

[1]. EU Joint Programme - Neurodegenerative Disease. What is neurodegenerative disease? JPND. 2015

[2]. Taipa R, Pinho J, Melo-Pires M. Clinicopathological correlations of the most common neurodegenerative dementias. Front Neurol. 2017 Jan;3:68.

[3]. Dalvi A. Alzheimer's Disease. Dis Mon. 2018 Dec;58(12):666-77.

[4]. Castellani RJ, Rolston RK, Smith MA. Alzheimer Disease. Dis Mon. 2016 Sep;56(9):484-546.

[5]. Xiaoting S, Jin L, Ling P. Review of drugs for Alzheimer's disease. Drug Discov Ther. 2017;6(6):285-90. 
[6]. Mu Y, Gage FH. Adult hippocampal neurogenesis and its role in Alzheimer's disease. Mol Neurodegener. 2016 Jan;6:85.

[7]. Dong S, Duan Y, Hu Y, Zhao Z. Advances in the pathogenesis of Alzheimer's disease: a reevaluation of amyloid cascade hypothesis. TransI Neurodegener. BioMed Central Ltd; 2018 Jan 21;1(1):18.

[8]. Galimberti D, Scarpini E. Disease-modifying treatments for Alzheimer's disease. Ther Adv Neurol Disord. 2018;4(4):203-16.

[9]. Chu LW. Alzheimer's disease: early diagnosis and treatment. Hong Kong Med J. 2018;18(3):228-37.

[10]. Alzheimer's Disease International. Relatório sobre a Doença de Alzheimer no Mundo. 2018.

[11]. Sosa-Ortiz AL, Acosta-Castillo I, Prince MJ. Epidemiology of Dementias and Alzheimer's Disease. Arch Med Res. Elsevier Inc; 2017;43(8):600-8.

[12]. Chen JH, Lin KP, Chen YC. Risk Factors for Dementia. J Formos Med Assoc. Formosan Medical Association \& Elsevier; 2017;108(10):754-64.

[13]. World Health Organization. Dementia: a public health priority. World Health Organization. Reino Unido; 2017. Novas abordagens no desenvolvimento de fármacos modificadores da Doença de Alzheimer 64

[14]. Associação Alzheimer Portugal. Plano Nacional de Intervenção Alzheimer. 2016.

[15]. Fiţa IG, Enciu A-M, Stănoiu BP. New insights on Alzheimer's disease diagnostic. Rom J Morphol Embryol. 2016 Jan;52(3 Suppl):975-9.

[16]. Anderson SH. Alzheimer Disease [Em linha]. Medscape. 2015 [Consult. 2015 Mar 23]. Disponível na Internet: http://emedicine.medscape.com/article/113481 7- overview

[17]. Morris JK, Honea RA, Vidoni ED, Swerdlow RH, Burns JM. Is Alzheimer's disease a systemic disease? Biochim Biophys Acta. 2015 Sep;1842(9):1340-9.

[18]. Lemere CA. Immunotherapy for Alzheimer's disease: hoops and hurdles. Mol Neurodegener. Molecular Neurodegeneration; 2016;8(1):36.

[19]. Perl DP. Neuropathology of Alzheimer's Disease. Mt Sinai J Med. 2016;77(1):32- 42.

[20]. Serrano-Pozo A, Frosch MP, Masliah E, Hyman BT. Neuropathological Alterations in Alzheimer Disease. Cold Spring Harb Perspect Med. 2018;1(1):1- 24. 